\title{
Adolescents violents : mieux vaut prévenir que punir
}

\section{Adolescent violence: prevent rather than punish}

\author{
R.E. Tremblay \\ (C) Springer-Verlag France 2010
}

«Au lieu d'envisager les phénomènes sociaux comme des problèmes scientifiques, on marche à l'aventure en se confiant à son étoile. "

Claude Bernard (1871)

La violence physique chez les adolescents et les jeunes adultes suscite de vives préoccupations dans toutes les sociétés. Le risque d'être arrêté et trouvé coupable d'un acte criminel est en effet plus élevé à la fin de l'adolescence et au début de l'âge adulte qu'à tout autre moment de la vie dans tous les pays, et ce, depuis qu'on tient des statistiques criminelles [15]. Au cours des 40 dernières années, des centaines d'études épidémiologiques et développementales, réalisées par des chercheurs de différentes disciplines, ont tenté de mieux comprendre comment de mignons petits enfants deviennent des adolescents violents qui ne cessent de faire les manchettes et d'alimenter les débats politiques et idéologiques [19,21].

Les études épidémiologiques montrent très clairement que le facteur de risque le plus important est d'ordre biologique : le sexe ! En effet, la très grande majorité des adolescents violents sont de sexe masculin. Les statistiques des tribunaux en France sont éloquentes, à peine un meurtrier sur dix est de sexe féminin. Les études épidémiologiques n'arrivent généralement pas à étudier les facteurs de risque de la violence physique chez les adolescentes et les jeunes femmes adultes

R.E. Tremblay $(\bowtie)$

University College Dublin, Woodview House, Belfield,

Dublin 4, Ireland

e-mail : tremblar@grip.umontreal.ca

Université de Montréal, boulevard Édouard-Montpetit,

2900 Montréal, Québec H3T 1J4, Canada

Inserm (U669),

université Paris-Sud-Paris-XI, université Paris-Descartes-Paris-V, maison de Solenn, 97, boulevard de Port-Royal,

F-75679 Paris cedex 14, France

Ce texte est une adaptation d'un texte paru dans http://www. enfant-encyclopedie.com/fr-ca/agressivite-enfant/selon-lesexperts.html. parce qu'elles sont trop peu nombreuses. Ces mêmes études avec les adolescents et les jeunes hommes montrent que les principaux facteurs associés à la délinquance juvénile violente sont : la surveillance inadéquate des garçons par les parents, la dislocation de la famille, l'influence négative des pairs et la pauvreté $[13,20]$.

L'explication traditionnelle des mécanismes qui conduisent aux comportements violents a été résumée dans un rapport d'experts de l'Académie des sciences des États-Unis en 1993 : « les comportements agressifs et violents sont des réponses apprises par la frustration, ils peuvent aussi être appris en tant qu'instruments destinés à atteindre des buts, et l'apprentissage se produit en observant des modèles de ce type de comportement. Ces modèles peuvent être observés dans la famille, chez les pairs, ailleurs dans le quartier, dans les médias de masse ou dans la pornographie violente » [16].

Si l'agression physique est apprise en observant les modèles au sein des familles, dans les quartiers et chez les pairs, on peut poser les questions suivantes :

- à quel âge commence cet apprentissage ?

- La fréquence des agressions physiques augmente-t-elle à la suite de l'exposition à des modèles de comportement agressif?

- Pourquoi les filles n'apprennent pas aussi bien ?

- Quand et comment peut-on prévenir le développement des agressions physiques?

\section{Résultats des recherches récentes}

\section{Recherche qui débute à l’école primaire}

Jusqu'à tout récemment, la plupart des études sur les agressions physiques ont porté sur les adolescents et sur les adultes. Les études longitudinales réalisées à partir de grands échantillons d'enfants d'école primaire ont conduit à des résultats inattendus : la fréquence des agressions physiques diminue entre le moment où les enfants commencent l'école primaire et celui où ils terminent l'école secondaire. Le 
phénomène se présente autant chez les filles que chez les garçons, bien que la fréquence des agressions physiques est systématiquement plus basse chez les filles, particulièrement à l'adolescence. Cette baisse de la fréquence des agressions avec l'âge a été observée dans les années 1980 et 1990 au Canada, en Nouvelle-Zélande et aux États-Unis, au moment où le taux d'homicides était en augmentation $[5,19,20]$.

Cette diminution de la fréquence des actes d'agression physique avec l'âge était inattendue du point de vue de la théorie de l'apprentissage social de l'agression, puisqu'en vieillissant les enfants étaient exposés à un nombre toujours croissant de modèles d'agression physique. Des études longitudinales ont également montré qu'il est fort peu probable qu'un adolescent se mette tout à coup à présenter de sérieux problèmes d'agression physique à l'adolescence s'il n'avait pas ce problème à l'enfance $[3,4,12]$.

Ces découvertes mènent évidemment à une autre question : si la fréquence maximum d'utilisation des agressions physiques chez la plupart des enfants est au début de l'école primaire, à quel âge les enfants apprennent-ils à agresser physiquement? Jusqu'à très récemment, peu de chercheurs s'étaient penchés sur l'agression physique avant l'arrivée à l'école, sans doute pour trois bonnes raisons :

- les conséquences des agressions physiques perpétrées par un adolescent de 18 ans sont généralement plus dramatiques que celles des agressions physiques perpétrées par un enfant de trois ans ;

- la théorie de l'apprentissage social de l'agression nous a menés à croire que les enfants apprennent à agresser pendant leurs années à l'école parce qu'ils sont plus exposés aux modèles d'agression que les enfants d'âge préscolaire ;

- il est plus facile pour les chercheurs d'observer et d'interviewer les enfants d'âge scolaire.

\section{Recherche qui débute pendant la petite enfance}

Quelques études longitudinales menées en Amérique du Nord et en Europe, au cours des dix dernières années, ont suivi les enfants depuis la naissance et ont renversé notre opinion sur le développement de l'agression physique. Ces études montrent que si les enfants apprennent effectivement à agresser physiquement en observant des modèles, l'apprentissage se fait sans doute entre le début et la fin des 24 premiers mois après la naissance, parce que la plupart des mères rapportent que leur enfant a recouru à une forme ou à une autre d'agression physique avant la fin de la deuxième année de vie $[1,22]$. Cela dit, il y a des différences importantes quant à la fréquence des agressions physiques chez les jeunes enfants $[9,23]$. Ces études montrent, en effet, qu'une majorité d'enfants ont occasionnellement recours à l'agression physique, qu'une minorité y a recours beaucoup moins souvent que la majorité et qu'une autre minorité utilise ces agressions beaucoup plus souvent que la majorité. Beaucoup d'enfants d'âge préscolaire sont orientés vers des cliniques pour des problèmes de comportement, principalement parce qu'ils utilisent fréquemment des agressions physiques [11].

Ces études longitudinales sur le développement des agressions physiques pendant les années préscolaires ont montré que la fréquence de ces actes d'agression augmente chez la plupart des enfants jusqu'à environ trois ans et demi pour diminuer par la suite de manière constante [9]. Cependant, les différences entre les sexes sont déjà présentes au début de la vie. Moins de filles que de garçons atteignent les niveaux de fréquence les plus élevés, les filles ont tendance à réduire la fréquence de leurs actes d'agression plus tôt dans la vie, et l'écart entre les garçons et les filles atteint son maximum à l'adolescence [6].

Il est rassurant de constater que la majorité des humains apprennent au cours de l'enfance à gérer leurs émotions et à ne pas recourir à l'agression physique pour régler un problème. Cependant, ces études longitudinales montrent que la minorité des enfants (de 5 à $10 \%$ ) qui continuent à manifester des niveaux élevés d'agression physique sont rejetés par les autres enfants et sont les plus à risque de se livrer à des comportements de violence physique pendant l'adolescence $[2,5,12]$.

Il est à noter que si la fréquence des agressions physiques diminue à partir de la troisième ou de la quatrième année après la naissance, la fréquence des gestes d'agression indirects (ex. : propos désobligeants à l'insu de la personne visée) augmente de façon importante entre quatre et sept ans, et que les filles ont tendance à utiliser cette forme d'agression plus souvent que les garçons [8].

Paradoxalement, même si ce sont les garçons qui sont les plus à risque de violence physique, les principaux facteurs de risque de la violence des garçons se retrouvent chez les mères. En effet, mis à part le faible revenu, les principaux facteurs de risque d'avoir de sérieux problèmes d'agression au cours de la petite enfance sont le niveau d'éducation de la mère, la fréquence de ses problèmes de comportement à l'adolescence, une première grossesse en bas âge et la consommation de tabac pendant la grossesse [20]. Les études qui prennent en compte les facteurs génétiques et environnementaux suggèrent de multiples interactions gène-environnement, dont des effets importants des environnements prénatal et postnatal sur l'expression des gènes $[18,20]$.

En résumé, contrairement aux croyances traditionnelles, les enfants n'ont pas besoin d'observer des modèles d'agression physique pour apprendre à agresser, pas plus qu'ils n'ont besoin d'apprendre à faire une crise de colère. Ainsi, plutôt que d'apprendre à recourir à l'agression physique par l'intermédiaire de leur environnement, les enfants des humains apprennent à ne pas recourir à l'agression physique 
grâce à leur environnement. Les enfants qui ne font pas ces apprentissages d'alternatives à l'agression physique à l'âge où la majorité le font se voient rejetés par les autres et sont à haut risque de maintenir ces comportements jusqu'à l'adolescence, c'est-à-dire au moment où leur croissance physique les a rendus beaucoup plus dangereux pour leur entourage.

\section{Implications pour la prestation de services et pour l'élaboration des politiques}

Ces résultats ont plusieurs implications importantes pour la prévention de la violence physique à tous les âges et pour le soutien à donner aux parents et aux enfants dès le début de la vie.

Premièrement, parce que c'est à la petite enfance que les enfants apprennent à utiliser des alternatives à l'agression physique, c'est à cette période que l'on doit soutenir ceux qui ont des difficultés à apprendre ces alternatives, autrement ils seront à haut risque de devenir des agresseurs physiques chroniques à l'école primaire et secondaire. Les problèmes de développement de la communication verbale suivent la même logique. Ils apparaissent à la petite enfance, et l'absence de soutien intensif à cette période aura des conséquences désastreuses avec l'âge. D'ailleurs, les enfants qui ont des problèmes de comportement à la petite enfance ont plusieurs autres problèmes, dont le développement du langage.

Deuxièmement, étant donné les indications très claires de transmission intergénérationnelle des difficultés à contrôler l'expression de ses émotions, il est probable qu'un soutien des jeunes familles à risque dès la première grossesse aura des effets bénéfiques à long terme sur les comportements violents, comme sur le développement du langage, le succès à l'école et la santé en général de tous les enfants des parents qui ont eux-mêmes une longue histoire de difficultés d'adaptation [20]. Le fait que les garçons soient largement plus à risque de violence physique chronique, alors que ce sont les caractéristiques des mères qui sont les facteurs de risque les plus importants, conduit à la conclusion qu'il faut aider les jeunes filles qui sont en difficulté d'adaptation, même si elles ne sont pas violentes, parce qu'elles sont à haut risque de reproduire une nouvelle génération de garçons violents et de filles en difficulté sérieuse d'adaptation. La première grossesse d'une jeune fille qui a échoué à l'école et qui vit en situation de pauvreté devrait attirer au moins autant de ressources des services publics que les ressources investies pour un adolescent violent. D'ailleurs, les résultats de l'étude de Côté et al. [7] publiée dans ce numéro et de quelques essais cliniques randomisés célèbres vont dans ce sens $[14,17]$.
Ces conclusions rejoignent les nombreux travaux sur les origines développementales de plusieurs maladies physiques tels les problèmes cardiovasculaires, l'obésité et certaines formes de cancer. Il est clair que le développement foetal, particulièrement dans une perspective épigénétique, prépare le fotus à vivre dans un certain type d'environnement. Le développement de tous les organes (incluant le cerveau) des fœtus de mères mal alimentées, stressées et en mauvaise santé les prépare à vivre dans un environnement adverse [10]. De plus, ces conditions de vie adverse se maintiennent généralement durant la petite enfance. Il est probable que le soutien intensif et à long terme des jeunes femmes enceintes qui ont une longue histoire d'adversité aura des effets bénéfiques à long terme, autant pour la santé physique que pour la santé mentale de la mère et de l'enfant.

Finalement, comme l'agression physique est une réaction spontanée que les humains apprennent à contrôler au cours de l'enfance, nous sommes tous à risque d'y recourir de nouveau si nous nous retrouvons dans une situation difficile qui semble ne pouvoir se régler que par la force physique. C'est ce mécanisme par défaut qui explique probablement pourquoi tant de crimes violents sont commis par des gens qui n'ont pas une histoire d'agression physique chronique et pourquoi tant de conflits intrafamiliaux, entre groupes ethniques ou religieux, entre classes sociales et nations, conduisent à la violence physique. Cette violence des « gens normaux » existe bel et bien, mais elle est différente de la violence des individus qui ont des problèmes d'agression physique chronique parce qu'ils n'ont pas fait l'apprentissage du contrôle de cette forme extrême de communication pendant leur enfance.

Les politiques qui favorisent une éducation de qualité pendant la jeune enfance ont depuis longtemps réduit l'incidence des cas de violence chronique de même que le niveau général d'agression physique au sein de nos sociétés modernes [19]. Pour le bien-être de tous, il importe d'en faire bénéficier les parents et les enfants moins fortunés qui ont besoin d'un soutien particulier. Mais, il faut aussi des politiques qui visent à maintenir des environnements empreints de paix partout dans la société pour prévenir que des réactions agressives primitives ne percent la mince couche de civilité que la majorité des humains acquièrent en vieillissant.

Remarque : ce texte est une adaptation d'un texte paru dans http://www.enfant-encyclopedie.com/fr-ca/agressiviteenfant/selon-les-experts.html

\section{Références}

1. Alink LRA, Mesman J, Van Zeijil J, et al (2006) The early childhood aggression curve: development of physical aggression in 10 to 50-month-old children. Child Dev 77(4):954-66 
2. Barker ED, Boivin M, Brendgen M, et al (2008) Predictive validity and early predictors of peer-victimization trajectories in preschool. Arch Gen Psychiatry 65:1185-92

3. Barker ED, Séguin JR, White HR, et al (2007) Developmental trajectories of male physical violence and theft: relations to neurocognitive performance. Arch Gen Psychiatry 64(5):592-9

4. Brame B, Nagin DS, Tremblay RE (2001) Developmental trajectories of physical aggression from school entry to late adolescence. J Child Psychol Psychiatry 42(4):503-12

5. Broidy LM, Nagin DS, Tremblay RE, et al (2003) Developmental trajectories of childhood disruptive behaviors and adolescent delinquency: a six-site, cross-national study. Dev Psychol 39(2):222-45

6. Côté SM (2007). Sex differences in physical and indirect aggression: a developmental perspective. Eur J Crim Policy Res 13(3-4):183-200

7. Côté SM, Boivin M, Daniel DS, et al (2007) The role of maternal education and nonmaternal care services in the prevention of children's physical aggression problems. Arch Gen Psychiatry 64(11):1305-12

8. Côté SM, Vaillancourt T, Barker ED, et al (2007) The joint development of physical and indirect aggression: predictors of continuity and change during childhood. Dev Psychopathol 19(1): $37-55$

9. Côté SM, Vaillancourt T, Leblanc JC, et al (2006) The development of physical aggression from toddlerhood to pre-adolescence: a nation wide longitudinal study of Canadian children. J Abnorm Child Psychol 34(1):68-82

10. Gluckman PD, Hanson MA, Cooper C, et al (2008) Effect of in utero and early-life conditions on adult health and disease. N Engl J Med 359:61-73

11. Keenan K, Wakschlag LS (2000) More than the terrible twos: the nature and severity of behavior problems in clinic-referred preschool children. J Abnorm Child Psychol 28(1):33-46

12. Nagin D, Tremblay RE (1999) Trajectories of boys' physical aggression, opposition, and hyperactivity on the path to physi- cally violent and nonviolent juvenile delinquency. Child Dev 70(5):1181-96

13. Nagin DS, Tremblay RE (2001) Parental and early childhood predictors of persistent physical aggression in boys from kindergarten to high school. Arch Gen Psychiatry 58(4):389-94

14. Olds D, Henderson CR, Cole R, et al (1998) Long-term effects of nurse home visitation on children's criminal and antisocial behavior: fifteen-year follow-up of a randomised controlled trial. JAMA 280(14):1238-44

15. Quetelet A (1833). Recherches sur le penchant au crime aux différents âges. Bruxelles: M. Hayez, 2nd edition

16. Reiss AJ Jr, Roth JA (eds) (1993) National Research Council (US). Panel on the understanding and control of violent behavior. Understanding and preventing violence, vol 1. National Academy Press, Washington DC

17. Schweinhart L, Montie J, Xiang Z, et al (2005) Lifetime effects: The High/Scope Perry Preschool study through age 40., vol. 14, Monographs of the High/Scope Educational Research Foundation, Ypsilanti, MI: High/Scope Press

18. Szyf M, Weaver I, Provençal N, et al (2009) Epigenetics and behaviour. In: Tremblay RE, van Aken M, Koop W (eds) Development and prevention of behaviour problems: from genes to social policy. Psychology Press, New York

19. Tremblay RE (2008) Prévenir la violence dès la petite enfance. Éditions Odile Jacob, Paris

20. Tremblay RE (2010) Developmental origins of disruptive behaviour problems: the 'original sin' hypothesis, epigenetics and their consequences for prevention. J Child Psychol Psychiatry [Epub ahead of print]

21. Tremblay RE, Hartup WW, Archer J (eds) (2005) Developmental origins of aggression. Guilford Press, New York NY

22. Tremblay RE, Japel C, Pérusse D, et al (1999). The search for the age of "onset" of physical aggression: Rousseau and Bandura revisited. Crim Behav Ment Health 9(1):8-23

23. Tremblay RE, Nagin DS, Séguin JR, et al (2004) Physical aggression during early childhood: trajectories and predictors. Pediatrics 114(1):e43-e50 